\title{
In ricordo di Monia
}

Durante la gestazione di questo numero, Monia Andreani, nostra collega di Redazione, è venuta improvvisamente a mancare.

Vogliamo qui brevemente ricordarne la persona, la figura di intellettuale e l'impegno civile. Monia rimane viva anche nella mente e nel cuore di coloro che l'hanno conosciuta, o semplicemente letta.

La rivista Prisma dedicherà al suo ricordo un'apposita iniziativa nella prossima programmazione editoriale.

I curatori del numero 